\title{
Erratum: Resummed photon spectrum from dark matter annihilation for intermediate and narrow energy resolution
}

\author{
M. Beneke, ${ }^{a}$ A. Broggio, ${ }^{b, c}$ C. Hasner, ${ }^{a}$ K. Urban ${ }^{a}$ and M. Vollmann ${ }^{a}$ \\ ${ }^{a}$ Physik Department T31, Technische Universität München, \\ James-Franck-Straße 1, D-85748 Garching, Germany \\ ${ }^{b}$ Università degli Studi di Milano-Bicocca, \\ Piazza della Scienza 3, I-20126 Milano, Italy \\ ${ }^{c}$ INFN - Sezione di Milano-Bicocca, \\ Piazza della Scienza 3, I-20126 Milano, Italy \\ E-mail: alessandro.broggio@unimib.it, caspar.hasner@tum.de, \\ kai.urban@tum.de, martin.vollmann@tum.de
}

ERRATUM TO: JHEP08(2019)103

ARXIV EPRINT: 1903.08702

In the course of working on the extension [2] of ref. [1] to Higgsino dark matter annihilation, we became aware of two mistakes and a number of typos in this article. The first correction implies that all absolute annihilation cross sections reported or shown in ref. [1] must be multiplied by two. The second correction concerns a missing term in some soft function components. This correction affects many equations, but has a negligible effect on the numerical results. In the following we provide a complete list of corrections.

Overall normalization of the annihilation cross section. Eqs. (2.38) and (4.1) miss a factor of two on the right-hand side and the correct equations read

$$
\begin{gathered}
\frac{d\left(\sigma v_{\mathrm{rel}}\right)}{d E_{\gamma}}=2 \sum_{I, J} S_{I J} \Gamma_{I J}\left(E_{\gamma}\right)=2 \sum_{I, J} S_{I J} \sum_{i, j=1,2} C_{i}(\mu) C_{j}^{*}(\mu) \gamma_{I J}^{i j}\left(E_{\gamma}, \mu\right), \\
\langle\sigma v\rangle=2 \times\left\{\mathbf{S}_{(\mathbf{0 0})(\mathbf{0 0})}[\sigma v]_{(00)(00)}+2 \operatorname{Re}\left[\mathbf{S}_{(\mathbf{0 0})(+-)}[\sigma v]_{(00)(+-)}\right]\right. \\
\left.+\mathbf{S}_{(+-)(+-)}[\sigma v]_{(+-)(+-)}\right\} .
\end{gathered}
$$

The factor of 2 is necessary in the method-2 computation of the Sommerfeld effect [3] for the annihilation of two identical particles to compensate for the method-2 factor $1 /(\sqrt{2})^{n_{\text {id }}}$, which appears in $\gamma_{I J}^{i j}\left(E_{\gamma}, \mu\right)$. In consequence the absolute cross sections shown in the upper panel of figure 3 and in both panels of figures 4 and 5 should also be multiplied by two. Since figures 3 and 4 display the most important results of the paper, we provide the corrected versions on the following pages. 
Soft function coefficients. A contribution from the integral $I_{v v}^{\text {real }}$ (defined in appendix C.2) was missed. We list the corrected versions of the equations, which change due to this omission.

$$
\begin{aligned}
W_{(+-)(+-)}^{22}(\omega, \mu, \nu)= & \delta(\omega)+\frac{\hat{\alpha}_{2}}{4 \pi}\left[\delta(\omega)\left(-8 \ln \frac{m_{W}}{\mu}-16 \ln \frac{m_{W}}{\mu} \ln \frac{m_{W}}{\nu}\right)\right. \\
& \left.+\left[\frac{1}{\omega}\right]_{*}^{\left[m_{W}\right]}\left(-6 \ln \left(\frac{m_{W}^{2}+\omega^{2}}{m_{W}^{2}}\right)-2 \frac{\omega^{2}}{m_{W}^{2}+\omega^{2}}+8 \ln \frac{\mu^{2}}{m_{W}^{2}}\right)\right], \\
\hat{W}_{(+-)(+-)}^{22}\left(\omega, \mu_{s}, \nu\right)= & {\left[1+\frac{\hat{\alpha}_{2}}{4 \pi}\left(\left(-16 \ln \frac{m_{W}}{\mu_{s}} \partial_{\eta}\right)-8 \ln \frac{m_{W}}{\mu_{s}}\right)\right] \frac{e^{-\gamma_{E} \eta}}{\Gamma(\eta)} \frac{1}{\omega}\left(\frac{\omega}{\nu}\right)^{\eta} } \\
& +\frac{\hat{\alpha}_{2}}{4 \pi}[-6 F(\omega)-2 P(\omega)] .
\end{aligned}
$$

We further specify the Laplace transform and inverse Laplace transform of the new structure:

$$
\begin{aligned}
\mathcal{L}\left\{\frac{\omega}{m_{W}^{2}+\omega^{2}}\right\} & =\cos \left(m_{W} s\right) \operatorname{ci}\left(m_{W} s\right)-\sin \left(m_{W} s\right) \operatorname{si}\left(m_{W} s\right) \equiv \tilde{Q}(s) \\
P(\omega) & \equiv \mathcal{L}^{-1}\left[\left(\frac{\kappa}{\nu}\right)^{\eta} \tilde{Q}\left(e^{-\gamma_{E}} / \kappa\right)\right] \\
& =\left(\frac{e^{-\gamma_{E}}}{\nu}\right)^{\eta} \frac{\omega^{1+\eta}}{m_{W}^{2} \Gamma(2+\eta)}{ }_{3} F_{2}\left(1,1, \frac{3}{2} ; 1+\frac{\eta}{2}, \frac{3}{2}+\frac{\eta}{2} ;-\frac{\omega^{2}}{m_{W}^{2}}\right) .
\end{aligned}
$$

The consequences of this correction for the numerical results in section 4 are very minor. The sentences specifying the relative effect of resummation on p. 37 should read:

"It is also apparent that the different levels of resummation successively reduce the theoretical uncertainty considerably, from $17 \%$ at LL, to $8 \%$ at NLL and $1 \%$ at NLL' at $m_{\chi}=2 \mathrm{TeV}$. Numerically, for the two mass values $m_{\chi}=$ $2 \mathrm{TeV}(10 \mathrm{TeV})$ the ratio to the Sommerfeld-only rate is $0.641_{-0.097}^{+0.115}\left(0.402_{-0.077}^{+0.096}\right)$ at LL, $0.707_{-0.054}^{+0.054}\left(0.463_{-0.033}^{+0.032}\right)$ at NLL and $0.667_{-0.006}^{+0.007}\left(0.435_{-0.004}^{+0.005}\right)$ at NLL'".

Furthermore the corrected eqs. (4.3), (4.4) are

$$
\begin{aligned}
\langle\sigma v\rangle= & 2 \times[\underbrace{\mathbf{3 4 . 2 4 6} \times(1.2799)}_{\sim 3 \%}+\underbrace{2 \operatorname{Re}[\mathbf{4 2 . 1 0 0} \times(-0.9173+5.7918 i)]}_{\sim-5 \%} \\
& +\underbrace{\mathbf{5 1 . 7 5 5} \times(29.907)}_{\sim 102 \%}] \times 10^{-28} \mathrm{~cm}^{3} / \mathrm{s}=3.0289 \times 10^{-25} \mathrm{~cm}^{3} / \mathrm{s} \\
\langle\sigma v\rangle= & 2 \times[\underbrace{\mathbf{1 . 1 3 4 5} \times(1.2440)}_{\sim 19 \%}+\underbrace{2 \operatorname{Re}[\mathbf{0 . 3 5 1 0 3} \times(-0.9553+7.7861 i)]}_{\sim-9 \%} \\
& +\underbrace{\mathbf{0 . 1 0 8 6 1 \times ( 6 2 . 7 8 8 )}}_{\sim 90 \%}] \times 10^{-27} \mathrm{~cm}^{3} / \mathrm{s}=1.5120 \times 10^{-26} \mathrm{~cm}^{3} / \mathrm{s} .
\end{aligned}
$$


In addition to the modifications in individual terms, this includes the overall factor of 2 correction discussed above. Figures 3 and 4 provided in this erratum have been generated with the corrected soft function but except at the low end of the $m_{\chi}$ range, only the factor of two change is visible.

Due to the new term, the list of abbreviations defined after (5.7) should be extended to include

$$
\kappa_{R}=\kappa_{R}\left(x_{\gamma}\right)=\frac{1}{2} \ln \left(1+x_{\gamma}^{2}\right) .
$$

$\kappa_{R}$ exhibits the same scaling with $E_{\text {res }}^{\gamma}$ as $\lambda_{R}$ in table 1 . The following equations in section 5 and appendix $\mathrm{C}$ change and the corrected versions are as follows:

$$
\begin{aligned}
& c_{(+-)(+-)}^{\operatorname{int}(1,0)}=\frac{1}{4}\left(\frac{19}{6}-\frac{11}{3} s_{W}^{2}\right) l_{\mu^{2}}-\frac{73}{18}+\frac{5 \pi^{2}}{12}+\frac{1}{4} z_{\gamma}+l_{R}^{2}-\frac{19}{24} l_{R}-\frac{3}{2} \lambda_{R}-\frac{1}{2} \kappa_{R}, \\
& {[\sigma v]_{(+-)(+-)}^{\text {nrw 1-loop }}=[\sigma v]_{(+-)(+-)}^{\text {int 1-loop }}+[\sigma v]_{(+-)(+-)}^{\text {tree }} \frac{\hat{\alpha}_{2}}{\pi}\left[\frac{3}{2} \lambda_{R}\left(\frac{2 E_{\text {res }}^{\gamma}}{m_{W}}\right)\right.} \\
& \left.+\frac{1}{2} \kappa_{R}\left(\frac{2 E_{\mathrm{res}}^{\gamma}}{m_{W}}\right)\right] \\
& c_{(+-)(+-)}^{\operatorname{int}(2,2)}=\frac{1}{4}\left(-\frac{3}{4}\right)\left[\frac{19}{3}-\frac{11}{3} s_{W}^{2}\right] l_{\mu^{2}}+ \\
& +\frac{4489}{2304}-\frac{37 \pi^{2}}{48}-\frac{3}{16} z_{\gamma}+\frac{9}{8} \lambda_{R}+\frac{3}{8} \kappa_{R}+l_{R}-\frac{1}{4} l_{R}^{2}, \\
& \frac{[\sigma v]_{(+-)(+-)}^{\mathrm{nrw}}-[\sigma v]_{(+-)(+-)}^{\mathrm{int}}}{[\sigma v]_{(+-)(+-)}^{\text {tree }}}=\frac{\hat{\alpha}_{2}}{\pi}\left[\frac{3}{2} \lambda_{R}+\frac{1}{2} \kappa_{R}\right]+\frac{\hat{\alpha}_{2}^{2}}{\pi^{2}}\left[-\frac{L^{4}}{32}+\left(\frac{19}{144}-l_{R}\right) L^{3}+\mathcal{O}\left(L^{2}\right)\right] \text {, } \\
& W_{(00)(00)}^{22}(\omega, \mu, \nu)=\frac{\hat{\alpha}_{2}}{4 \pi}\left[\frac{1}{\omega}\right]_{*}^{\left[m_{W}\right]}\left(8 \ln \left(\frac{m_{W}^{2}+\omega^{2}}{m_{W}^{2}}\right)-8 \frac{\omega^{2}}{m_{W}^{2}+\omega^{2}}\right), \\
& W_{(00)(+-)}^{22}(\omega, \mu, \nu)=W_{(+-),(00)}^{22 *}(\omega, \mu, \nu) \\
& =\frac{\hat{\alpha}_{2}}{4 \pi}\left[\delta(\omega)(8-8 \pi i) \ln \frac{m_{W}}{\mu}+\left[\frac{1}{\omega}\right]_{*}^{\left[m_{W}\right]}\right. \\
& \left.\times\left(4 \ln \left(\frac{m_{W}^{2}+\omega^{2}}{m_{W}^{2}}\right)+4 \frac{\omega^{2}}{m_{W}^{2}+\omega^{2}}\right)\right], \\
& W_{(+-)(+-)}^{22}(\omega, \mu, \nu)=\delta(\omega)+\frac{\hat{\alpha}_{2}}{4 \pi}\left[\delta(\omega)\left(-8 \ln \frac{m_{W}}{\mu}-16 \ln \frac{m_{W}}{\mu} \ln \frac{m_{W}}{\nu}\right)\right. \\
& \left.+\left[\frac{1}{\omega}\right]_{*}^{\left[m_{W}\right]}\left(-6 \ln \left(\frac{m_{W}^{2}+\omega^{2}}{m_{W}^{2}}\right)-2 \frac{\omega^{2}}{m_{W}^{2}+\omega^{2}}+8 \ln \frac{\mu^{2}}{m_{W}^{2}}\right)\right], \\
& \hat{W}_{(00)(00)}^{22}\left(\omega, \mu_{s}, \nu\right)=\frac{\hat{\alpha}_{2}}{4 \pi}[8 F(\omega)-8 P(\omega)],
\end{aligned}
$$



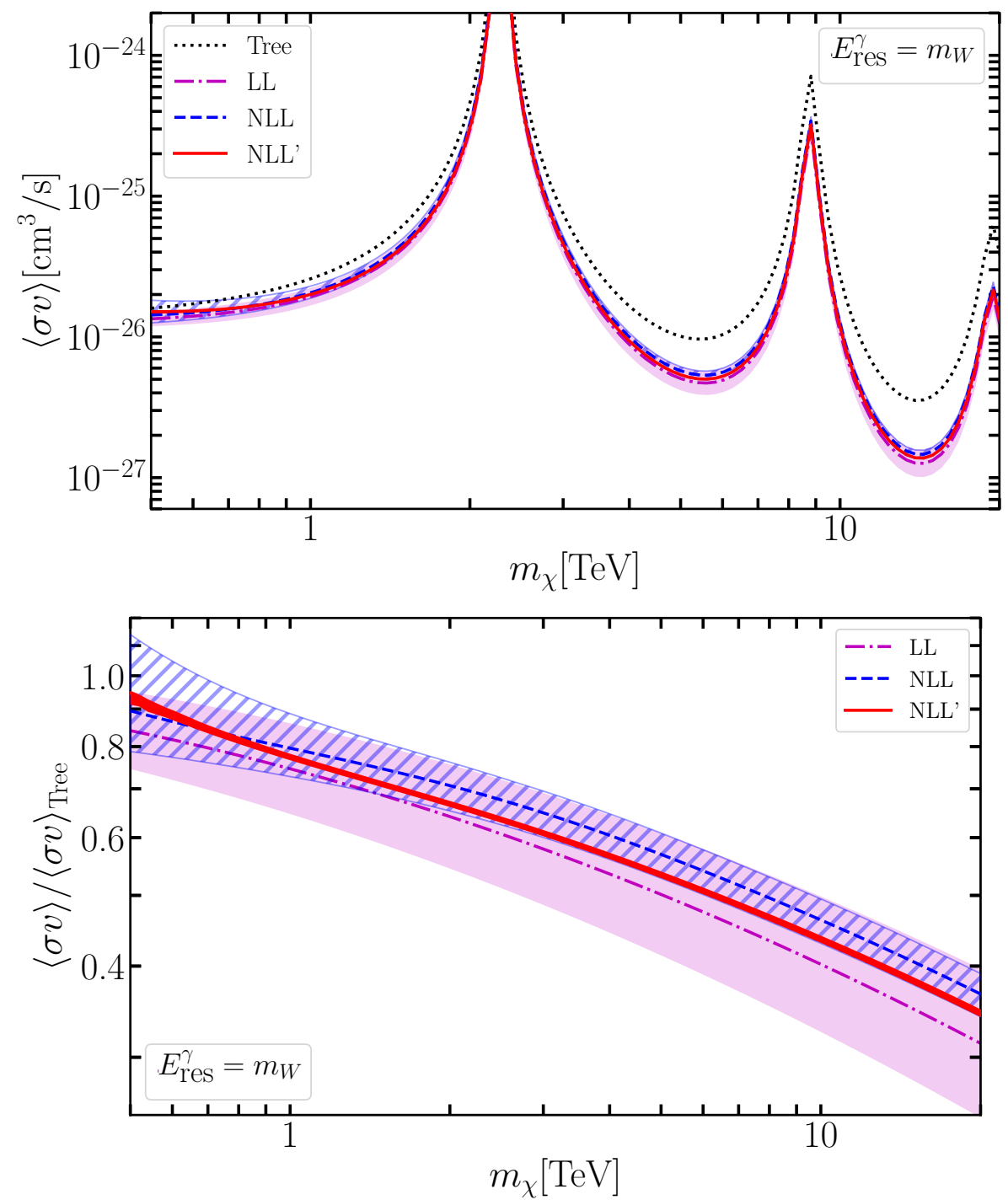

Figure 3. Integrated photon energy spectrum within $E_{\mathrm{res}}^{\gamma}$ from the endpoint $m_{\chi}$ in the tree (Sommerfeld only) and LL, NLL, NLL' resummed approximation. The energy resolution is set to $E_{\mathrm{res}}^{\gamma}=m_{W}$. The shaded/hatched bands show the scale variation of the respective approximation as described in the text. For the NLL' result the theoretical uncertainty is given by the thickness of the red line.

$$
\begin{aligned}
\hat{W}_{(00)(+-)}^{22}\left(\omega, \mu_{s}, \nu\right)= & \hat{W}_{(+-)(00)}^{22 *}\left(\omega, \mu_{s}, \nu\right) \\
= & {\left[\frac{\hat{\alpha}_{2}}{4 \pi}(8-8 \pi i) \ln \frac{m_{W}}{\mu_{s}}\right] \frac{e^{-\gamma_{E} \eta}}{\Gamma(\eta)} \frac{1}{\omega}\left(\frac{\omega}{\nu}\right)^{\eta}+\frac{\hat{\alpha}_{2}}{4 \pi}[4 F(\omega)+4 P(\omega)], } \\
\hat{W}_{(+-)(+-)}^{22}\left(\omega, \mu_{s}, \nu\right)= & {\left[1+\frac{\hat{\alpha}_{2}}{4 \pi}\left(\left(-16 \ln \frac{m_{W}}{\mu_{s}} \partial_{\eta}\right)-8 \ln \frac{m_{W}}{\mu_{s}}\right)\right] \frac{e^{-\gamma_{E} \eta}}{\Gamma(\eta)} \frac{1}{\omega}\left(\frac{\omega}{\nu}\right)^{\eta} } \\
& +\frac{\hat{\alpha}_{2}}{4 \pi}[-6 F(\omega)-2 P(\omega)] .
\end{aligned}
$$



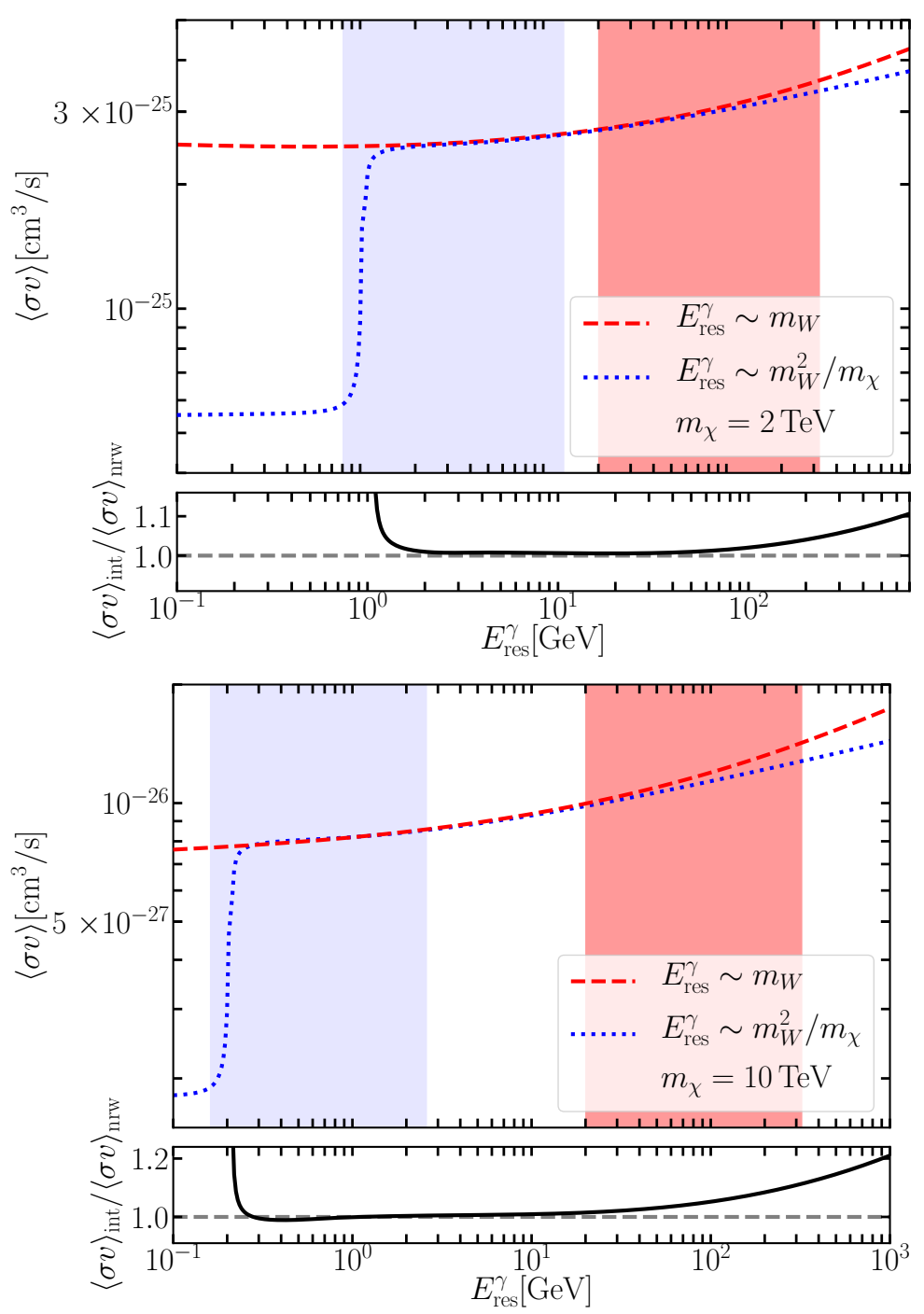

Figure 4. Annihilation cross sections plotted as function of $E_{\mathrm{res}}^{\gamma}$. The blue-dotted line shows the cross section for the narrow resolution computed in [4]. The red-dashed line shows the intermediate resolution cross section. The light-grey (blue) area represents the region of validity for the narrow resolution case and the dark-grey (red) area represents the region of validity for the intermediate resolution case. The ratio of the intermediate to narrow resolution annihilation cross section $\langle\sigma v\rangle_{\text {int }} /\langle\sigma v\rangle_{\text {nrw }}$ is added below each plot. The results are shown for DM masses of $m_{\chi}=2 \mathrm{TeV}$ (upper plot) and $m_{\chi}=10 \mathrm{TeV}$ (lower plot).

In appendix E, eqs. (E.10), (E.11) and (E.12) require correction. To allow for the contribution from the new term $\kappa_{R}\left(x_{\gamma}\right)$ it is convenient to generalize the definitions of the integrals in (E.5) and (E.6) to

$$
\begin{aligned}
& \varphi_{f_{R}}=\varphi_{f_{R}}\left(x_{\gamma}\right) \equiv \int_{0}^{x_{\gamma}} \frac{\mathrm{d} y}{y}\left[f_{R}\left(x_{\gamma}-y\right)-f_{R}\left(x_{\gamma}\right)\right] \\
& \vartheta_{f_{R}}=\vartheta_{f_{R}}\left(x_{\gamma}\right) \equiv \int_{0}^{x_{\gamma}} \mathrm{d} y \frac{\ln (y)}{y}\left[f_{R}\left(x_{\gamma}-y\right)-f_{R}\left(x_{\gamma}\right)\right] .
\end{aligned}
$$


For reasons of brevity, we will not explicitly repeat the expansion coefficients in (E.10), (E.11) and (E.12) already given in [1]. Instead, we denote them by $c_{I J \text {,old }}^{\operatorname{int}(n, m)}$ and simply add the new terms that originate from the previously omitted $\omega /\left(m_{W}^{2}+\omega^{2}\right)$-terms in the soft functions. Coefficients that do not change are not repeated. The corrected expressions are:

$$
\begin{aligned}
& c_{(00)(00)}^{\operatorname{int}(1,0)}=c_{(00)(00), \text { old }}^{\text {int }(1,0)}-2 \kappa_{R} \\
& c_{(00)(00)}^{\operatorname{int}(2,2)}=c_{(00)(00), \text { old }}^{\operatorname{int}(2,2)}+\frac{3 \kappa_{R}}{2} \\
& c_{(00)(00)}^{\operatorname{int}(2,1)}=c_{(00)(00), \text { old }}^{\text {int }(2,1)}-2 \kappa_{R} l_{R}-\frac{125}{24} \kappa_{R}-2 \varphi_{\kappa_{R}} \\
& c_{(00)(00)}^{\operatorname{int}(2,0)}=c_{(00)(00), \text { old }}^{\operatorname{int}(2,0)}+l_{\mu_{s}}\left(-2 \kappa_{R} l_{\nu_{s}}+4 \kappa_{R} l_{R}+\frac{91 \kappa_{R}}{12}+4 \varphi_{\kappa_{R}}\right) \\
& +l_{\mu} \kappa_{R}\left(-\frac{19}{6}+\frac{11}{6} \hat{s}_{W}^{2}\right)-2 \kappa_{R} l_{R}^{2}+l_{R}\left(\frac{19 \kappa_{R}}{12}-4 \varphi_{\kappa_{R}}\right) \\
& -\frac{\kappa_{R} z_{\gamma}}{2}+\left(\frac{1}{9}+\frac{\pi^{2}}{6}\right) \kappa_{R}+\frac{19 \varphi_{\kappa_{R}}}{12}-4 \vartheta_{\kappa_{R}} \\
& c_{(+-)(00)}^{\operatorname{int}(1,0)}=c_{(+-)(00), \text { old }}^{\operatorname{int}(1,0)}+\kappa_{R} \\
& c_{(+-)(00)}^{\operatorname{int}(2,2)}=c_{(+-)(00), \text { old }}^{\operatorname{int}(2,2)}-\frac{3}{4} \kappa_{R} \\
& c_{(+-)(00)}^{\operatorname{int}(2,1)}=c_{(+-)(00), \text { old }}^{\operatorname{int}(2,1)}+\kappa_{R} l_{R}+\frac{125}{48} \kappa_{R}+\varphi_{\kappa_{R}} \\
& c_{(+-)(00)}^{\operatorname{int}(2,0)}=c_{(+-)(00), \text { old }}^{\operatorname{int}(2,0)}+\left[l_{\nu_{s}} \kappa_{R}-2 l_{R} \kappa_{R}-\frac{91}{24} \kappa_{R}-2 \varphi_{\kappa_{R}}\right] l_{\mu_{s}} \\
& +\left(\frac{19}{12}-\frac{11}{12} \hat{s}_{W}^{2}\right) \kappa_{R} l_{\mu}+\kappa_{R} l_{R}^{2}+\left(-\frac{19}{24} \kappa_{R}+2 \varphi_{\kappa_{R}}\right) l_{R} \\
& +\left(-\frac{1}{18}-\frac{\pi^{2}}{12}+\frac{z_{\gamma}}{4}\right) \kappa_{R}-\frac{19}{24} \varphi_{\kappa_{R}}+2 \vartheta_{\kappa_{R}} \\
& c_{(+-)(+-)}^{\operatorname{int}(1,0)}=c_{(+-)(+-), \text {old }}^{\operatorname{int}(1,0)}-\frac{1}{2} \kappa_{R} \\
& c_{(+-)(+-)}^{\operatorname{int}(2,2)}=c_{(+-)(+-), \text {old }}^{\operatorname{int}(2,2)}+\frac{3}{8} \kappa_{R} \\
& c_{(+-)(+-)}^{\operatorname{int}(2,1)}=c_{(+-)(+-), \text {old }}^{\operatorname{int}(2,1)}-\frac{1}{2} \kappa_{R} l_{R}-\frac{125}{96} \kappa_{R}-\frac{1}{2} \varphi_{\kappa_{R}} \\
& c_{(+-)(+-)}^{\operatorname{int}(2,0)}=c_{(+-)(+-), \text {old }}^{\operatorname{int}(2,0)}+l_{\mu_{s}}\left[-\frac{1}{2} \kappa_{R} l_{\nu_{s}}+\kappa_{R} l_{R}+\frac{91}{48} \kappa_{R}+\varphi_{\kappa_{R}}\right] \\
& +\left(-\frac{19}{24}+\frac{11}{24} \hat{s}_{W}^{2}\right) \kappa_{R} l_{\mu}-\frac{1}{2} \kappa_{R} l_{R}^{2}+l_{R}\left(\frac{19}{48} \kappa_{R}-\varphi_{\kappa_{R}}\right) \\
& +\left(\frac{1}{36}+\frac{\pi^{2}}{24}-\frac{z_{\gamma}}{8}\right) \kappa_{R}+\frac{19}{48} \varphi_{\kappa_{R}}-\vartheta_{\kappa_{R}}
\end{aligned}
$$




\section{Further corrections.}

- In eq. (2.10) the $\chi$ field on the left in the $\chi \chi$ bilinears should be transposed.

- In section 2.1.3 we derive the annihilation operators. In the paragraph below (2.35) we state that there are three spin- 0 and four spin- 1 operators left. This statement should instead say that there are four spin-0 and three spin-1 operators left.

- In $(2.53)$, a factor of $1 /(2 \pi)$ should be added to the last line such that $(2.53)$ changes to

$$
\begin{aligned}
-g_{\mu \mu^{\prime}}^{\perp} J^{X V}\left(p^{2}, m_{W}\right) & =\frac{1}{\pi} \operatorname{Im}\left[-g_{\mu \mu^{\prime}}^{\perp} i \mathcal{J}^{X V}\left(p^{2}, m_{W}\right)\right] \\
& \equiv \frac{1}{\pi} \operatorname{Im}\left[i \int d^{4} x e^{i p \cdot x}\left\langle 0\left|\mathbf{T}\left\{\mathcal{A}_{\perp c, \mu^{\prime}}^{X}(x) \mathcal{A}_{\perp c, \mu}^{V}(0)\right\}\right| 0\right\rangle\right] \\
& =\frac{1}{2 \pi} \int d^{4} x e^{i p \cdot x}\left\langle 0\left|\mathcal{A}_{\perp c, \mu^{\prime}}^{X}(x) \mathcal{A}_{\perp c, \mu}^{V}(0)\right| 0\right\rangle .
\end{aligned}
$$

The same factor of $1 /(2 \pi)$ should be added to (B.28). In (2.59), $2 \pi$ needs to be multiplied to lines 2, 3 and 4 of the equation. Furthermore, (2.61) should read

$$
\begin{aligned}
\gamma_{I J}^{i j}\left(E_{\gamma}\right)= & \frac{1}{4} \frac{1}{2 \pi m_{\chi}}\left\langle\xi_{0}^{c \dagger} \Gamma_{j}^{\mu \nu} \xi_{0}\right\rangle^{*}\left\langle\xi_{0}^{c \dagger} \Gamma_{i, \mu \nu} \xi_{0}\right\rangle \\
& \times Z_{\gamma}^{33} \int d \omega J^{X V}\left(4 m_{\chi}\left(m_{\chi}-E_{\gamma}-\omega / 2\right), m_{W}\right) W_{I J, V 3 X 3}^{i j}(\omega),
\end{aligned}
$$

and the result of (2.62) is 4 instead of 8 . The numerical results are not affected by these corrections.

- The comparison between the resummation schemes in section 5.3 was incorrect and should instead read

$$
\begin{gathered}
\frac{[\sigma v]_{(00)(00)}^{\text {Res.Sc.I }}-[\sigma v]_{(00)(00)}^{\text {Res.Sc.II }}}{[\sigma v]_{(00)(00)}^{\text {tree }}}=\frac{\hat{\alpha}_{2}^{2}}{\pi^{2}} 4 l_{R}\left(\varphi_{\lambda_{R}}-\varphi_{\kappa_{R}}\right), \\
\frac{[\sigma v]_{(00)(+-)}^{\text {Res.ScI }}-[\sigma v]_{(00)(+-)}^{\text {Res.Sc.II }}}{[\sigma v]_{(00)(+-)}^{\text {tree }}}=\frac{\hat{\alpha}_{2}^{2}}{\pi^{2}} 2 l_{R}\left(\varphi_{\lambda_{R}}+\varphi_{\kappa_{R}}\right), \\
\frac{[\sigma v]_{(+-)(+-)}^{\text {Res.Sc.I }}-[\sigma v]_{(+-)(+-)}^{\text {Res.Sc.II }}}{[\sigma v]_{(+-)(+-)}^{\text {tree }}}=-\frac{\hat{\alpha}_{2}^{2}}{\pi^{2}} l_{R}\left(3 \varphi_{\lambda_{R}}+\varphi_{\kappa_{R}}\right) .
\end{gathered}
$$

Open Access. This article is distributed under the terms of the Creative Commons Attribution License (CC-BY 4.0), which permits any use, distribution and reproduction in any medium, provided the original author(s) and source are credited. 


\section{References}

[1] M. Beneke, A. Broggio, C. Hasner, K. Urban and M. Vollmann, Resummed photon spectrum from dark matter annihilation for intermediate and narrow energy resolution, JHEP 08 (2019) 103 [arXiv: 1903.08702] [INSPIRE].

[2] M. Beneke, C. Hasner, K. Urban and M. Vollmann, Precise yield of high-energy photons from Higgsino dark matter annihilation, JHEP 03 (2020) 030 [arXiv:1912.02034] [INSPIRE].

[3] M. Beneke, C. Hellmann and P. Ruiz-Femenia, Non-relativistic pair annihilation of nearly mass degenerate neutralinos and charginos III. Computation of the Sommerfeld enhancements, JHEP 05 (2015) 115 [arXiv: 1411.6924] [INSPIRE].

[4] M. Beneke, A. Broggio, C. Hasner and M. Vollmann, Energetic $\gamma$-rays from TeV scale dark matter annihilation resummed, Phys. Lett. B $\mathbf{7 8 6}$ (2018) 347 [arXiv:1805. 07367] [INSPIRE]. 